\title{
Thermosensitive hydrogel based on chitosan and its derivatives containing medicated nanoparticles for transcorneal administration of 5-fluorouracil
}

This article was published in the following Dove Press journal:

International Journal of Nanomedicine

16 January 2017

Number of times this article has been viewed

\author{
Angela Fabiano' \\ Ranieri Bizzarri² \\ Ylenia Zambito' \\ 'Department of Pharmacy, University \\ of Pisa, ${ }^{2} \mathrm{NEST}$, Istituto Nanoscienze \\ CNR (CNR-NANO) and Scuola \\ Normale Superiore, Pisa, Italy
}

\begin{abstract}
A thermosensitive ophthalmic hydrogel (TSOH) - fluid at $4^{\circ} \mathrm{C}$ (instillation temperature), semisolid at $35^{\circ} \mathrm{C}$ (eye temperature), which coupled the dosing accuracy and administration ease of eyedrops with the increased ocular bioavailability of a hydrogel - was prepared by gelling a chitosan hydrochloride $(\mathrm{ChHCl})$ solution $(27.8 \mathrm{mg} / \mathrm{mL})$ medicated with $1.25 \mathrm{mg} / \mathrm{mL}$ 5-fluorouracil (5-FU) with $\beta$-glycerophosphate $0.8 \mathrm{mg} / \mathrm{mL}$. Polymer mixtures, where Ch was partially $(10 \%, 15 \%$, or $20 \%)$ replaced by quaternary ammonium-chitosan conjugates (QA-Ch) or thiolated derivatives thereof, were also used to modulate 5-FU-release properties of TSOH. Also, Ch-based nanoparticles (NPs; size after lyophilization and redispersion $341.5 \pm 15.2 \mathrm{~nm}$, polydispersity $0.315 \pm 0.45$, $\zeta$-potential $10.21 \mathrm{mV}$ ) medicated with $1.25 \mathrm{mg} / \mathrm{mL} 5$-FU prepared by ionotropic cross-linking of Ch with hyaluronan were introduced into TSOH. The 5-FU binding by TSOH polymers in the sol state was maximum with plain Ch (31.4\%) and tended to decrease with increasing QA presence in polymer mixture. 5-FU release from TSOH with or without NPs was diffusion-controlled and linear in $\sqrt{t}$. The different TSOH polymers were compared on a diffusivity basis by comparing the slopes of $\sqrt{ }$ t plots. These showed a general decrease with NP-containing TSOH, which was the most marked with the TSOH, where Ch was $20 \%$ replaced by the derivative QA-Ch50. This formulation and that not containing NP were instilled in rabbits and the 5-FU transcorneal penetration was measured by analyzing the aqueous humor. Both TSOH solutions increased the area under the curve (0-8 hours) 3.5 times compared with the plain eyedrops, but maximum concentration for the NP-free TSOH was about $0.65 \mu \mathrm{g} / \mathrm{mL}$, followed by a slow decline, while the NP-containing one showed a plateau $(0.25-0.3 \mu \mathrm{g} / \mathrm{mL})$ in a time interval of $0.5-7$ hours. This is ascribed to the ability of this TSOH to control drug release to a zero order and that of NPs to be internalized by corneal cells.
\end{abstract}

Keywords: thermosensitive hydrogels, chitosan nanoparticles, chitosan multifunctional derivatives, 5-fluorouracil, ocular delivery

\section{Introduction}

Ocular drug delivery is one of the more challenging tasks for pharmaceutical scientists. The unique structure of the eye restricts the entry of drug molecules at the required site of action. More than $90 \%$ of the marketed ophthalmic formulations are produced in the form of eyedrops, which are washed away from the eye by various mechanisms, ie, lachrymation, tear dilution, and turnover, resulting in low ocular bioavailability of drugs. Moreover, the human cornea, comprising the epithelium, substantia propria, and endothelium, restricts the entry of drug molecules. ${ }^{1}$ As a result of the action of these factors, less than $5 \%$ of the administered drug enters the eye.
Correspondence: Ylenia Zambito Department of Pharmacy, University of Pisa, 33 Via Bonanno, Pisa 56|26, Italy Tel +390502219657

Fax +39050 2219660

Email ylenia.zambito@unipi.it 
Colloidal carriers have been widely exploited in the field of drug-delivery science, because they provide more selective targeting with sustained release of molecules at the desired site and can be used in the treatment of diseases affecting the anterior and the posterior segments of the eye. An ideal therapy requires selective targeting of an active agent for various eye diseases, such as choroidal neovascularization, diabetic retinopathy, and solid tumors. The retina does not possess any lymphatic system or angiogenesis, so this part of the eye has similar features to a solid tumor with enhanced permeability and retention effects., ${ }^{2,3}$ Delivery of a drug via nanotechnology mainly pursues the following three objectives: 1) enhancing drug permeation, 2) controlling drug release, and 3) drug targeting. ${ }^{3}$ Another interesting approach to ocular drug delivery is the use of hydrogel systems. Hydrogels are hydrophilic polymers able to swell in water or aqueous solvents. Preformed hydrogels do not allow accurate or reproducible administration of drugs, and after administration they often produce blurred vision, crusting of eyelids, and lachrymation. Therefore, the most promising approach is the use of in situ-forming hydrogels that can be instilled as eyedrops and undergo immediate gelation when in contact with the eye. ${ }^{4}$ In situ-forming hydrogels provide a response to environmental changes, 5,6 indeed, they are liquid upon instillation and undergo phase transition in the ocular cul-de-sac to form viscoelastic gels. ${ }^{7,8}$ This class of biomaterials was first presented by Chenite et al. ${ }^{9}$ They described polymer-polyol salt aqueous solutions, which could be held liquid below room temperature and turned into monolithic gels at body temperature. In light of this information, in the present study, we felt it intriguing to combine the characteristics of a thermosensitive hydrogel with those of nanoparticles (NPs) to promote transcorneal penetration of 5-fluorouracil (5-FU). Among the various mucoadhesive polymers described in the literature, the cationic polymer chitosan $(\mathrm{Ch})$ has been considered the polymer of choice to prepare NPs and hydrogels, because of its positive charge and ability to interact intimately with the cornea and conjunctiva, which are negatively charged. In the hydrogel formulation, in some cases, small fractions of $\mathrm{Ch}$ were substituted with quaternary ammonium-Ch conjugates (QA-Ch) or thiolated derivatives thereof (QA-Ch-SH), ${ }^{10}$ because of their improved mucoadhesiveness and ability to promote transcorneal penetration. ${ }^{11}$ NPs medicated with 5-FU were lyophilized and dispersed in the systems in the sol state. The formulations were compared on the grounds of their affinity with the drug and in vitro drug-release properties, in order to select the system on which to carry out in vivo pharmacokinetic studies. To this end, such a system had to be liquid at manageably low temperatures, undergo an immediate gelation when in contact with the physiological temperature of the eye, and be suitable for in vivo administration to rabbits. 5-FU was chosen as the model drug, as it is the drug of choice for the treatment of ocular cancer. A $1 \%$ 5-FU solution is currently being used by ophthalmologists, which is a high concentration for ophthalmic application. Bioavailability in the anterior segment of the eye is less than $5 \%$ of the applied dose because of drainage, lachrymation, and poor retention at the absorption surface. ${ }^{12}$ The ability of the selected NP-containing hydrogel to enhance transcorneal penetration of 5-FU compared to traditional eyedrops and produce a sustained drug level in the aqueous humor was tested in this study.

\section{Materials and methods Materials}

5-FU, Ch of low molecular weight, $\beta$-glycerophosphate $(\beta-\mathrm{GP})$ disodium salt, and trehalose dehydrate were from Sigma-Aldrich (St Louis, MO, USA). ChHCl was prepared as previously described. ${ }^{13}$ Fluorescein isothiocyanate (FITC) was from Sigma-Aldrich. The QA-Ch conjugates QA-Ch50 and QA-Ch60 and thiolated derivatives QA-Ch50-SH and QA-Ch60-SH were synthesized from $\mathrm{Ch}$ according to Zambito et al. ${ }^{10}$ For the $\mathrm{Ch}$ derivatives, the figures 50 and 60 refer to the respective temperatures $\left({ }^{\circ} \mathrm{C}\right)$ at which the synthesis was carried out. Hyaluronic acid (viscometric molecular weight $470 \mathrm{kDa}$ ) was prepared as described by Zambito et al. ${ }^{10}$ Nylon 6.6 and cellulose membranes were soaked for at least 24 hours in water before use. All aqueous solutions/ dispersions were prepared with deionized water. All other chemicals and solvents were of reagent grade. FITC labeling of Ch was carried out as previously described. ${ }^{14-17}$

\section{Quantitative analysis of 5-FU}

Quantitative analysis of 5-FU was carried out by highperformance liquid chromatography (HPLC). The apparatus (PerkinElmer, Waltham, MA, USA) consisted of a series 200 pump, $20 \mu \mathrm{L}$ Rheodyne injector, ultraviolet detector, and Turbochrom Navigator HPLC software (PerkinElmer) for data integration. A Spheri-5 RP-18 $5 \mu \mathrm{m} 250 \times 4.6 \mathrm{~mm}$ column was used. The mobile phase (flow rate $0.6 \mathrm{~mL} / \mathrm{min}$ ) was $\mathrm{KH}_{2} \mathrm{PO}_{4} 0.05 \mathrm{M}$ triethylamine $0.1 \%$, and ultraviolet detection was set at $266 \mathrm{~nm}$. Standard curves were constructed by analyzing six standard drug solutions in the eluent. All standard curves produced on different days were linear $\left(r^{2}>0.998\right)$ in the concentration range $0.3-5 \mu \mathrm{g} / \mathrm{mL}$ (limit of quantification $0.2 \mu \mathrm{g} / \mathrm{mL}$, limit of detection $0.07 \mu \mathrm{g} / \mathrm{mL}$ ). The retention 
time was 7.5 minutes. The concentration of each unknown sample, analyzed by the aforementioned, was determined using a standard curve produced on the same day.

\section{Preparation of medicated NPs}

FITC-labeled or unlabeled Ch-based NPs medicated with 5-FU were self-assembled upon portion-wise addition of a $950 \mu \mathrm{L}$ volume of hyaluronic acid $(1 \mathrm{mg} / \mathrm{mL})$ and $5-\mathrm{FU}$ $(5.21 \mathrm{mg} / \mathrm{mL})$ in demineralized water to $5 \mathrm{~mL}$ of $1 \mathrm{mg} / \mathrm{mL}$ FITC-labeled or unlabeled $\mathrm{ChHCl}$ in demineralized water. The final 5-FU concentration in the NP dispersion was $1.25 \mathrm{mg} / \mathrm{mL}$, corresponding to the concentration contained in the commercial 5-FU eyedrops. Following preparation, the NPs were checked for size and $\zeta$-potential (Nano ZS90; Malvern Instruments, Malvern, UK). Their drug-entrapment efficiency (EE) was evaluated by subjecting the dispersion to centrifugation $\left(28,800 \mathrm{rpm}\right.$ for 1 hour at $\left.4^{\circ} \mathrm{C}\right)$ and analyzing the supernatant for the drug by HPLC. EE was expressed as follows:

$$
\mathrm{EE}=\left(\mathrm{M}_{\mathrm{t}}-\mathrm{M}_{\mathrm{s}}\right) / \mathrm{M}_{\mathrm{t}}
$$

where $M_{t}$ is the total drug used for the preparation and $\mathrm{M}_{\mathrm{s}}$ the mass found in the supernatant.

To obtain stable systems, the drug-loaded NP dispersions were lyophilized following the addition of $250 \mathrm{mg}$ of trehalose dehydrate as cryoprotectant (VirTis Advantage; thermal treatment step $-35^{\circ} \mathrm{C}$ for 180 minutes, drying cycle steps $-30^{\circ} \mathrm{C}$ for 360 minutes, $-10^{\circ} \mathrm{C}$ for 360 minutes, $10^{\circ} \mathrm{C}$ for 240 minutes, and $25^{\circ} \mathrm{C}$ for 180 minutes). The dispersions were able to be regenerated from the respective lyophilized products by adding $5 \mathrm{~mL}$ of water under gentle stirring.

\section{Preparation of thermosensitive hydrogels medicated with 5-FU}

Thermosensitive ophthalmic hydrogel (TSOH) was prepared by dissolving a mass of $500 \mathrm{mg}$ of $\mathrm{Ch}$, alone or mixed with different fractions $(10 \%, 15 \%$, or $20 \%)$ of $\mathrm{Ch}$ derivatives, namely, QA-Ch50, QA-Ch60, QA-Ch50-SH, or QA-Ch60-SH, in $18 \mathrm{~mL}$ of a $0.08 \mathrm{M} \mathrm{HCl}$ solution. The resulting solutions were kept under magnetic stirring at $4^{\circ} \mathrm{C}$, and an adequate volume of $\beta$-GP solution $(0.8 \mathrm{~g} / \mathrm{mL})$ was added dropwise. The resulting solution was heated to $35^{\circ} \mathrm{C}$, after which a sol-gel transition was organoleptically evident. Medicated hydrogels containing $1.25 \mathrm{mg} / \mathrm{mL} 5$-FU were able to be obtained by the aforementioned procedure. 5-FU only or lyophilized 5-FU-medicated NPs were added to the initial $\mathrm{Ch}$-based solution. The content in $\mathrm{Ch}$ and $\mathrm{Ch}$ derivative of
Table I Composition of TSOH tested

\begin{tabular}{llll}
\hline Code & $\begin{array}{l}\text { Concentration } \\
\text { of Ch }(\mathbf{m g} / \mathbf{m L})\end{array}$ & $\begin{array}{l}\text { Concentration } \\
\text { of Ch derivative } \\
(\mathbf{m g} / \mathbf{m L}, \%)\end{array}$ & $\begin{array}{l}\beta \text {-GP } \\
\text { amount } \\
(\mu \mathbf{L})\end{array}$ \\
\hline Ch & 27.8 & - & 476 \\
QA + QA10-Ch60 & 25 & $2.8,10$ & 400 \\
Ch + QA15-Ch60 & 23.6 & $4.2,15$ & 450 \\
Ch + QA20-Ch60 & 22.2 & $5.6,20$ & 450 \\
Ch + QA10-Ch60-SH & 25 & $2.8,10$ & 400 \\
Ch + QA15-Ch60-SH & 23.6 & $4.2,15$ & 425 \\
Ch + QA20-Ch60-SH & 22.2 & $5.6,20$ & 450 \\
Ch + QA10-Ch50 & 25 & $2.8,10$ & 400 \\
Ch + QA15-Ch50 & 23.6 & $4.2,15$ & 450 \\
Ch + QA20-Ch50 & 22.2 & $5.6,20$ & 450 \\
QA10-Ch50-SH & 25 & $2.8,10$ & 400 \\
QAI5-Ch50-SH & 23.6 & $4.2,15$ & 450 \\
Ch + QA20-Ch50-SH & 22.2 & $5.6,20$ & 476 \\
\hline
\end{tabular}

Abbreviations: TSOH, thermosensitive ophthalmic hydrogel; $\mathrm{Ch}$, chitosan; $\beta$-GP, $\beta$-glycerophosphate; QA, quaternary ammonium.

each formulation and the volume of $\beta$-GP necessary for its gelation are reported in Table 1 . The formulations were given the codes Ch + QA $x$-Chy or Ch + QA $x$-Chy-SH, where $x$ and $y$ are two figures: $x$ representing the fraction of $C h$ derivative in the polymer mixture $(10 \%, 15 \%$, or $20 \%)$ and $y$ the type of Ch derivative (50 or 60$)$.

\section{Confocal microscopy and image analysis}

To evaluate the possibility of redispersing lyophilized NP into hydrogel systems, the lyophilized FITC-labeled Ch-based NPs, prepared as described in the "Preparation of medicated NPs" section, were dispersed in the $\mathrm{Ch}+\mathrm{QA} 20-\mathrm{Ch} 50 \mathrm{TSOH}$ at room temperature and observed under confocal laser-scanning microscopy (TCS SP5, Leica Microsystems, Wetzlar, Germany). Images were captured at $21^{\circ} \mathrm{C}$ and $35^{\circ} \mathrm{C}$ with the system in liquid and gel states, respectively. Excitation wavelength was set at $488 \mathrm{~nm}(10-20 \mu \mathrm{W}$ power emission at objective), whereas emission was collected in the 500-550 nm range. Scanning speed was set at 400 lines per second for an image format of $512 \times 512$ pixels. A $63 \times 1.25$ numeric-aperture water objective was used, setting the pinhole aperture of the confocal system at 1 Airy unit.

\section{Dynamic dialysis studies}

A well-known procedure and underlying theory ${ }^{18,19}$ was used to assess reversible drug binding by $\mathrm{Ch}$ in fluid solution or the surface of the NPs in fluid dispersion. The diffusion cell, apparatus, and procedure were the same as those described in Bottari et al. ${ }^{18}$ These were used at a thermostat temperature of $35^{\circ} \mathrm{C}$. The dialysis membrane, permeable 
to the drug and impermeable to the polymers and NPs, was made of porous cellulose (cutoff $12.5 \mathrm{kDa}$ ). At $t=0$, $5 \mathrm{~mL}$ of medicated NPs, regenerated after lyophilization, were introduced in the donor compartment of the cell. Sink conditions were maintained in the receiving phase (100 mL of demineralized water). Drug transport across the membrane was assessed by analyzing the receiving phase at intervals for the drug by HPLC. At the end of each run, the donor phase was withdrawn from the cell and checked for particle size, as reported in the "Preparation of medicated NPs" section. Dialysis was also carried out, using the same procedure and membrane type, for the plain-drug solution (control) and this solution containing $0.25 \mathrm{mg} / \mathrm{mL}$ of $\mathrm{ChHCl}$ dissolved in water.

\section{Interrupted-dialysis studies}

Interrupted-dialysis experiments were carried out to gain information on drug release from NPs while concurrently checking data according to suggestions from Zambito et al. ${ }^{19}$ To this purpose, the dialysis, carried out as described in the "Dynamic dialysis studies" section, was stopped after an established time from the start and the drug fraction contained in each of the NP matrix, NP-dispersion medium, and acceptor medium had been determined. This procedure was repeated, running the dialysis for different times, to construct the plots of the drug fraction in each phase versus time. To determine the drug fraction in the dispersion medium and calculate that in the NP matrix, ultracentrifugation of the donor phase followed by analysis of the supernatant was carried out.

\section{Determination of drug-polymer interactions in $\mathrm{TSOH}$}

These interactions were determined as they could be determinant to in vivo drug residence in precorneal area, and thereby, to drug ocular bioavailability.

\section{Sol state}

The dynamic dialysis technique described in the "Dynamic dialysis studies" section was used at the temperature of $4^{\circ} \mathrm{C}$ to determine drug-polymer interactions for the TSOH systems in the sol state, the composition of which is listed in Table 1. At $t=0,5 \mathrm{~mL}$ of medicated formulation was introduced in the donor compartment of the cell. Sink conditions were maintained in the receiving phase $(100 \mathrm{~mL}$ of $\mathrm{HCl} 0.08 \mathrm{M}$ and $\beta$-GP). Drug transport across the membrane was assessed by analyzing the receiving phase at intervals for the drug. In the course of a typical day of this study, the control was run in the first place, followed by sequential testing of two TSOH systems, and finally the control was repeated, without disassembling the membrane between runs. With this procedure, the slopes of the two control runs were not significantly different (Student's $t$-test, $P<0.05$ ). This procedure allowed the daily, two-by-two determination of drug binding for the TSOH listed in Table 1. At the end of each run, the donor phase was withdrawn for particle-size checking. A fresh membrane was used each day.

\section{Gel state}

In this case, the phase-equilibration technique, already described in detail in the literature, ${ }^{20}$ was used. In brief, the apparatus consisted of six cells, each made of two hemicells, one of which in all cases contained a $0.2 \mathrm{~cm}$ thick layer $(2 \mathrm{~mL})$ of gel of known drug concentration $(1.25 \mathrm{mg} / \mathrm{mL})$ separated by a nonporous nylon 6.6 membrane (surface area $10.2 \mathrm{~cm}^{2}$ ) from $7 \mathrm{~mL}$ of drug solution in phosphate buffer (PB; pH 7.4, 0.13 M) contained in the other hemicell. The initial drug concentration in the PB-containing hemicells was different among the different cells and spanned a range supposed to encompass the unknown equilibrium concentration with the gel. Each gel-containing hemicell was tightly connected with a PB-containing one by a clamp, and at time 0 , the six assemblies were simultaneously shaken in a thermostatic water bath at $35^{\circ} \mathrm{C}$. In each cell, the drug tended to equilibrate between the gel and PB phases via the membrane. After an appropriate interval (6 hours), sufficient for a positive or negative concentration change in each solution in PB to be detectable, such a change was determined and plotted vs the corresponding initial concentration. The resulting plot allowed calculation of the bound-drug fraction.

\section{Studies on 5-FU release from TSOH}

5 -FU release from TSOH was carried out using a specially designed Teflon cell. The gel $(0.5 \mathrm{~mL}, 1.25 \mathrm{mg} / \mathrm{mL})$, containing (or not) medicated NPs, was introduced in the cylindrical cavity of the cell (diameter of the available area for diffusion $50 \mathrm{~mm}$ ). A porous cellulose membrane (cutoff $12.5 \mathrm{kDa}$ ), kept in place by a circular Teflon plate and O-ring gasket, was used to separate the gel from the receiving phase $(30 \mathrm{~mL}$ of PB, pH 7.4, 0.13 M). For use, the filled cell was placed in a jacketed beaker (internal height $11 \mathrm{~cm}$, internal diameter $10 \mathrm{~cm}$ ) connected with a constant-temperature bath and circulator set at $35^{\circ} \mathrm{C}$. At time 0 , the preequilibrated receiving phase was introduced in the beaker and stirred with a 300 rpm synchronous motor. Drug release was determined by analyzing the receiving phase at intervals. 


\section{In vivo studies}

For in vivo tests, four types of medicated ophthalmic drops were prepared, with the following compositions: 1) 5 -FU in PB (1.25 mg/mL, control, $310 \mathrm{mOsm}), 2)$ the thermosensitive Ch solution containing $1.25 \mathrm{mg} / \mathrm{mL} 5$-FU (210 mOsm), 3) the thermosensitive $\mathrm{Ch}+\mathrm{QA20-Ch50} \mathrm{solution} \mathrm{contain-}$ ing $1.25 \mathrm{mg} / \mathrm{mL} 5$-FU (367 mOsm), and 4) a dispersion of Ch-based NPs, medicated with $1.25 \mathrm{mg} / \mathrm{mL} 5-\mathrm{FU}$, in the thermosensitive $\mathrm{Ch}+\mathrm{QA20}-\mathrm{Ch} 50$ solution (416 mOsm). The eyedrops described in 1 had the same solvent and drug concentration as the commercial eyedrops.

Male New Zealand albino rabbits weighing 3-3.5 kg maintained under standard housing conditions were used. They were treated as prescribed in the Guide for the Care and Use of Laboratory Animals (revised 1985). All experiments were carried out under veterinary supervision, and the protocols were approved by the scientific ethics committee of the University of Pisa and the Italian Ministry of Education, Universities, and Research. The irritation, possibly caused in rabbit eyes by the ophthalmic drops, was evaluated by searching eyes for signs of conjunctival/corneal edema and/or hyperemia at the end of each in vivo experiment. ${ }^{21}$ For measurement of 5-FU intraocular penetration, preparations were made to $4^{\circ} \mathrm{C}$, then one drop $(50 \mu \mathrm{L})$ of each was instilled in the lower conjunctival sac. At preestablished times, rabbits were anesthetized by instilling one drop of Novesina ${ }^{\circledR}$, then $\sim 60 \mu \mathrm{L}$ of aqueous humor was aspirated from the anterior chamber. For analysis, each sample was mixed with acetonitrile (1:2), then it was centrifuged and the supernatant subjected to evaporation of the acetonitrile by heating the mixture to $50^{\circ} \mathrm{C}$ under an airstream. The aqueous product was then lyophilized. The resulting lyophilized product was redispersed in a volume of mobile phase corresponding to the volume of the sample of aqueous initially withdrawn and analyzed by HPLC. ${ }^{22}$

\section{Data treatment}

The statistical significance of linear patterns was evaluated by subjecting data to linear regression analysis and computing the relevant slope and intercept and coefficient of determination $\left(r^{2}\right)$. The number of data points for each linear regression is specified in the text, table title, or figure caption. The number of repeats for the values listed in the tables is specified in the table title. Each data point of in vitro and in vivo nonlinear plots is the mean \pm standard deviation of at least three repeats. The area under the curve (AUC) for the aqueous humor was calculated by the linear trapezoidal rule between 0 and 8 hours. The statistical methods described by Schoenwald et $\mathrm{al}^{23}$ were used to compare AUC values. The significance of differences was evaluated by Student's $t$-test $(P<0.05)$.

\section{Results and discussion Characteristics of medicated NPs immediately after preparation or regeneration after lyophilization}

Mean particle size, polydispersity index (PI), EE, and $\zeta$ potential values for freshly prepared NPs were $285.6 \pm 36.1 \mathrm{~nm}$, $0.32 \pm 0.05,36.6 \% \pm 3.1 \%$, and $10.32 \mathrm{mV}$, respectively, while the corresponding values for NPs regenerated from the lyophilized products were $341.5 \pm 15.2 \mathrm{~nm}, 0.31 \pm 0.04$, $34.9 \% \pm 2.9 \%$, and $10.21 \mathrm{mV}$. In all cases, the PI values pointed to sufficiently homogeneous size distribution according to the literature. ${ }^{24-26}$ These results altogether indicated that the NPs could effectively be protected from mechanical stress caused by ice crystallization by adding the cryoprotectant trehalose.

\section{Confocal microscopy and image analysis}

The confocal images for FITC-labeled medicated NPs dispersed in the $\mathrm{Ch}+\mathrm{QA} 20-\mathrm{Ch} 50 \mathrm{TSOH}$ are presented in Figure 1. Figure $1 \mathrm{~A}$ refers to the fluid dispersion at $21^{\circ} \mathrm{C}$ (room temperature), while Figure 1B refers to the semisolid hydrogel at $35^{\circ} \mathrm{C}$. Figure $1 \mathrm{C}$ is a magnified detail of Figure 1B. As can be seen, NPs are dispersed in either the liquid or in situ-forming hydrogel, the gelation of which did not involve any important modifications in NP-dispersion degree. Dimensional analysis of particles is reported in Figure 2. In particular, fluorescence was analyzed along a line equatorially crossing an NP, and the resulting fluorescence profile was fitted to a Gaussian function. ${ }^{27,28} \mathrm{~A}$ full width at half maximum of the Gaussian curve of about $360 \mathrm{~nm}$ was determined, which corresponded to the NP diameter and was not significantly different from that of freshly prepared NPs, reported in the "Characteristics of medicated NPs immediately after preparation or regeneration after lyophilization" section. The use of confocal microscopy was necessary, because it was not possible to determine the mean NP size by dynamic light scattering, due to the polymers in the solution interfering with the measurements.

\section{Drug-binding studies}

Dynamic dialysis data were used to assess reversible drug interactions with $\mathrm{Ch}$ in fluid solution or the surface of the NPs in fluid dispersion. According to the established theory on determination of drug-macromolecule binding by dynamic dialysis (or permeation), the data obtained in 

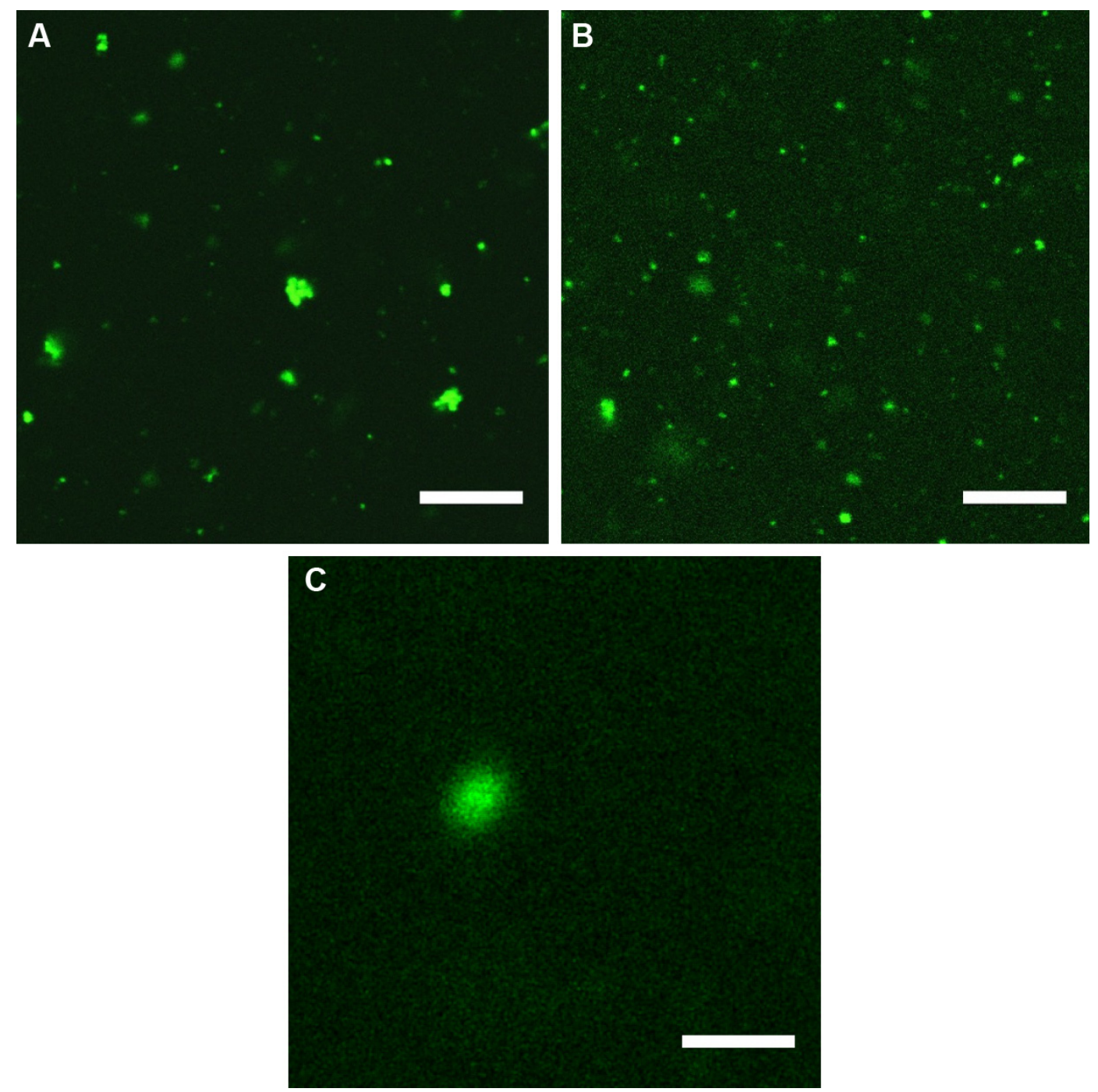

Figure I FITC-labeled NPs observed under confocal microscopy.

Notes: $(\mathbf{A}) 21^{\circ} \mathrm{C}$, dispersion in liquid state (scale $\left.10 \mu \mathrm{m}\right) ;(\mathbf{B}) 35^{\circ} \mathrm{C}$, dispersion in gel state (scale $\left.10 \mu \mathrm{m}\right)$; (C) $35^{\circ} \mathrm{C}$, dispersion in gel state (scale bar: I $\left.\mu \mathrm{m}\right)$. Abbreviations: FITC, fluorescein isothiocyanate; NPs, nanoparticles.

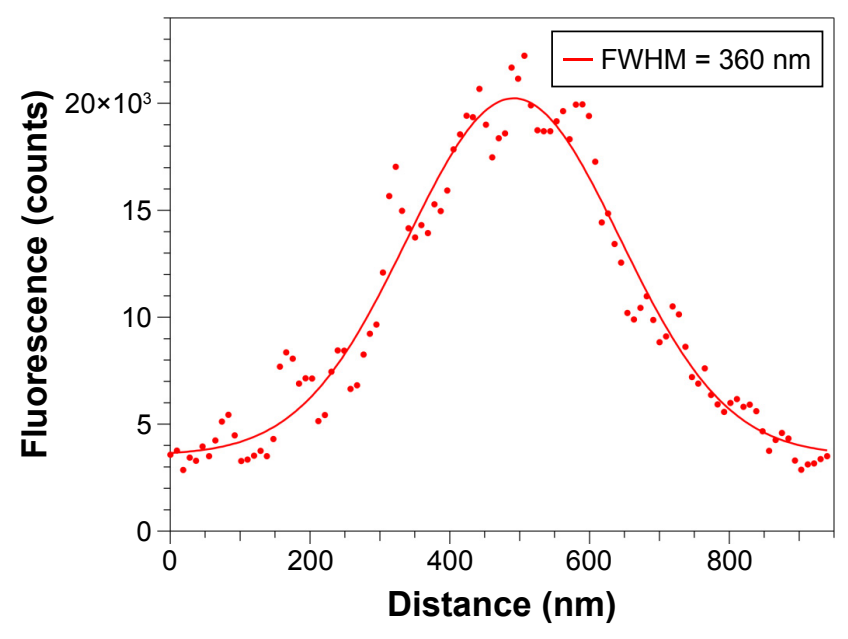

Figure 2 Confocal microscopy analysis of FITC-labeled NP size.

Abbreviations: FITC, fluorescein isothiocyanate; NP, nanoparticle; FWHM, full width at half maximum. this work were plotted according to the following equation, which was derived by applying Fick's first law to the transfer of a solute across a membrane under quasi-steady-state conditions: ${ }^{17-19,29}$

$$
\ln \left[\left(\mathrm{C}_{\mathrm{d}} / \mathrm{C}_{\mathrm{d}_{0}}\right) \times 100\right]=4.605-\mathrm{K}_{\mathrm{m}} \cdot \mathrm{F}_{\mathrm{f}} \cdot \mathrm{t}
$$

where $C_{d}$ is the drug concentration in the donor phase, $C_{d_{0}}$ is the drug concentration in the donor phase at time $0, \mathrm{~F}_{\mathrm{f}}$ is the noninteracting-to-total drug ratio in the donor phase, and $\mathrm{K}_{\mathrm{m}}$ is the dialysis-rate constant. Once $\mathrm{F}_{\mathrm{f}}$ is determined from the slope of the log-linear plot, the bound fraction can readily be calculated as $1-\mathrm{F}_{\mathrm{f}^{\prime}}$. The dialysis data for plain 5-FU (control), 5-FU-loaded NPs, and 5-FU in the presence of $\mathrm{Ch}$ in solution are plotted in Figure 3 as $\ln \left(\mathrm{C}_{\mathrm{d}} / \mathrm{C}_{\mathrm{d}_{0}}\right) \times 100$ versus t. 


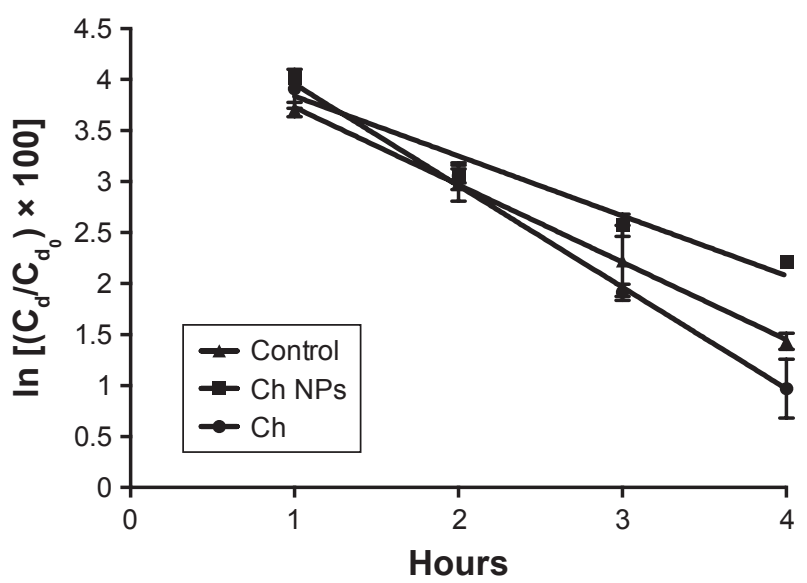

Figure 3 Dynamic dialysis data plotted according to Equation 2.

Notes: In the donor phase, 5 -FU $(1.25 \mathrm{mg} / \mathrm{mL})$ alone (control) or in the presence of $\mathrm{Ch}(0.25 \mathrm{mg} / \mathrm{mL})$ or Ch NPs (regenerated after lyophilization). Each data point is the mean \pm standard deviation of at least three repeats. Where not shown, the error bar is enclosed within the symbol.

Abbreviations: 5-FU, 5-fluorouracil; $\mathrm{Ch}$, chitosan; NPs, nanoparticles; $\mathrm{C}_{\mathrm{d}}$, drug concentration; $\mathrm{C}_{\mathrm{d}_{0}}, \mathrm{C}_{\mathrm{d}}$ at time 0 .

All plots are significantly linear, as shown by the respective $r^{2}$ values, namely, 0.999, 0.996, and 0.994. Such linearity indicates that in all cases Equation 2 was obeyed and $\mathrm{F}_{\mathrm{f}}$ was actually constant with varying $\mathrm{C}_{\mathrm{d}}$ and time at least over the time of dialysis. For the plain drug (control), $\mathrm{F}_{\mathrm{f}}=1$; therefore, according to Equation 2, the slope of the relative log-linear plot was equal to the dialysis-rate constant $\mathrm{K}_{\mathrm{m}}$. The slopes of the straight lines \pm standard deviation in Figure 3 were $0.841 \pm 0.029$ hours (control, $\mathrm{K}_{\mathrm{m}}$ ), $0.894 \pm 0.052$ hours $(5-\mathrm{FU}$ in Ch solution), and $0.543 \pm 0.019$ hours (5-FU in loaded NP dispersion). These data indicate a nonsignificant 5-FU-Ch binding in solution and a $35.4 \%$ bound fraction in the NP dispersion. From here, it was deduced that the drug fraction reversibly adsorbed on the NP surface was significantly higher than that reversibly interacting with $\mathrm{Ch}$ in solution. Since the NPs were made of mucoadhesive polymers, ${ }^{30}$ their binding to both the ocular surface and drug was encouraging in terms of bioavailability.

\section{Interrupted-dialysis studies}

There is still debate in the literature about the validity of dynamic dialysis as a method to obtain release data from NP systems. ${ }^{19}$ To make a further contribution to the discussion, a procedure was used in the present work based on interrupted dialysis, followed by ultracentrifugation of donor phase, as described in the "Materials and methods" section. Results from the kinetic analysis of phases are plotted in Figure 4. The drug fractions in the dispersion medium and NP matrix showed parallel decreasing patterns, suggesting a substantial thermodynamic equilibrium between these phases all along

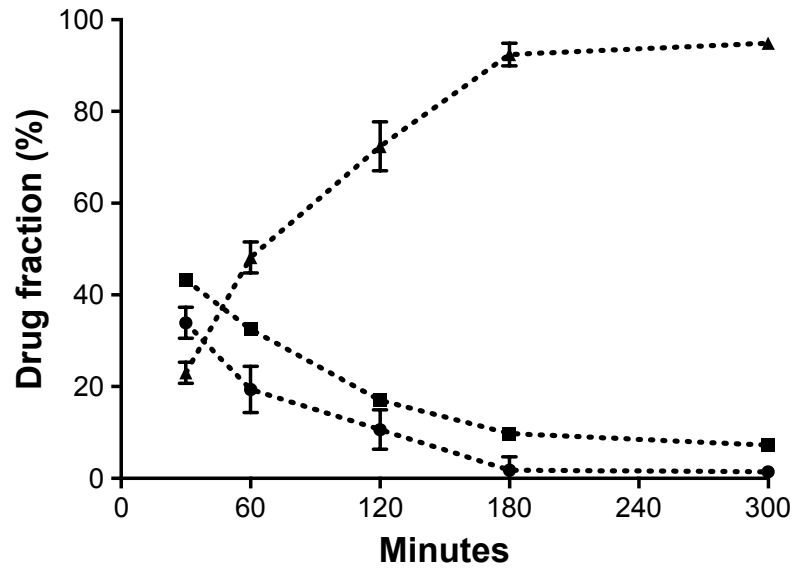

Figure 4 Interrupted dialysis studies: kinetic analysis of dialysis phases for 5-FUloaded NPs regenerated after lyophilization.

Notes: $\bullet$, NP matrix; $\boldsymbol{\square}, \mathrm{NP}$-dispersion medium; $\boldsymbol{\Delta}$, acceptor medium. Mean \pm standard deviation of three runs.

Abbreviations: 5-FU, 5-fluorouracil; NPs, nanoparticles.

the release process. Moreover, at whatever time of the process the drug fraction in the dispersion medium was significantly higher than in the NP matrix, suggesting that the former was no perfect sink for drug release from NPs. This indicates that such a process was under the control of the dialysis membrane. Indeed, if this were not the case, and drug release from NPs was NP-controlled, the 5-FU decrease in the NPdispersion medium would have been faster than in the NP matrix and the drug fraction in the former would soon have become lower than in the latter. The data support previous reports stating that dialysis is not always a safe method for studying drug release from nanoparticulate systems because of diffusional resistance to release, opposed by the dialysis membrane. ${ }^{19}$ Anyway, a piece of useful information still emerges from the data in Figure 4, ie, in such a medium as tear fluid, where sink conditions for the present NPs would be respected, the release of 5-FU from Ch-based NPs would be complete in less than 180 minutes.

\section{Determination of drug interactions with polymers used to prepare TSOH}

\section{Sol state}

Dynamic dialysis data were used to assess reversible drug interactions with the polymers used to prepare the hydrogel formulations in the sol state. The considerations reported in the "Drug-binding studies" section also apply to the present cases, only the experiments were carried out at $4^{\circ} \mathrm{C}$. The dialysis data for plain 5-FU (control) and those for the polymers used to prepare the hydrogels are reported as $\ln \left(\mathrm{C}_{\mathrm{d}} / \mathrm{C}_{\mathrm{d}_{0}}\right) \times 100$ versus $\mathrm{t}$ in Figure $5 \mathrm{~A}$ and $\mathrm{B}$. The linearity of plots appears from the figure. The significance of linearity 

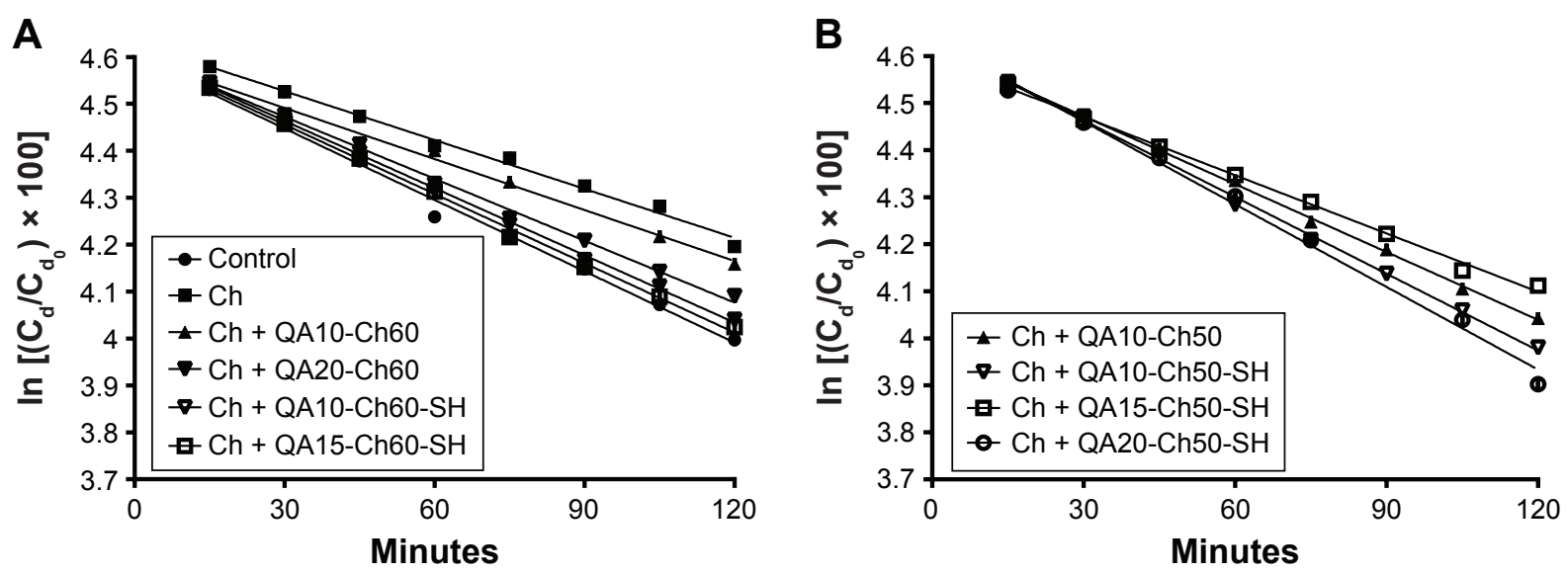

Figure 5 Dynamic dialysis data obtained at $4^{\circ} \mathrm{C}$, plotted according to Equation 2.

Notes: In the donor phase, 5-FU (I.25 mg/mL) alone (control) or in the presence of Ch or TSOH. The plots for the control (representative) and Ch are represented in A, and are not repeated in B. Some overlapping plots have been omitted for clarity. In all cases, the linearity of the plots is apparent.

Abbreviations: 5-FU, 5-fluorouracil; $\mathrm{Ch}$, chitosan; $\mathrm{TSOH}$, thermosensitive ophthalmic hydrogel; $\mathrm{QA}$, quaternary ammonium; $\mathrm{C}_{\mathrm{d}}$, drug concentration; $\mathrm{C}_{\mathrm{d}}$, $\mathrm{C}_{\mathrm{d}}$ at time 0 .

is confirmed by the respective $r^{2}$ values, listed in Table 2 . From the data, it appears that the partial substitution in the formulation of $\mathrm{Ch}$ with its derivatives generally weakened 5-FU interaction with the formulation polymers in the sol state to negligible values.

\section{Gel state}

In this case, drug-polymer interaction was studied by the phase-equilibration technique described in the previous "Gel state" section. The positive or negative concentration change in each solution in PB was plotted versus the correspondent

Table 2 Results of analysis of dynamic dialysis data obtained at $4^{\circ} \mathrm{C}$ for 5 -FU plotted in Figure 5 according to Equation 2

\begin{tabular}{|c|c|c|c|}
\hline Formulation & $\begin{array}{l}\text { Modulus of straight- } \\
\text { line slope }\left(\times 10^{3}\right) \pm \\
\text { SD }(n=8)(1 / \text { minutes })\end{array}$ & $r^{2}$ & $\begin{array}{l}\text { Interaction }^{a} \\
(\%)\end{array}$ \\
\hline Control & $5.06 \pm 0.75$ & 0.998 & - \\
\hline $\mathrm{Ch}$ & $3.47 \pm 0.23$ & 0.993 & 31.4 \\
\hline $\mathrm{Ch}+\mathrm{QA} 10-\mathrm{Ch} 60$ & $3.62 \pm 0.13$ & 0.995 & 28.5 \\
\hline $\mathrm{Ch}+\mathrm{QAI} 5-\mathrm{Ch} 60$ & $4.4 \pm 0.12$ & 0.997 & 13 \\
\hline $\mathrm{Ch}+\mathrm{QA} 20-\mathrm{Ch} 60$ & $4.77 \pm 0.07$ & 0.999 & 5.7 \\
\hline $\mathrm{Ch}+\mathrm{QA} I 0-\mathrm{Ch} 60-\mathrm{SH}$ & $4.4 \pm 0.18$ & 0.996 & 13 \\
\hline $\mathrm{Ch}+\mathrm{QA} / 5-\mathrm{Ch} 60-\mathrm{SH}$ & $4.92 \pm 0.1$ & 0.998 & 2.8 \\
\hline $\mathrm{Ch}+\mathrm{QA20}-\mathrm{Ch} 60-\mathrm{SH}$ & $5.14 \pm 0.09$ & 0.998 & NS \\
\hline $\mathrm{Ch}+\mathrm{QA} 10-\mathrm{Ch} 50$ & $4.8 I \pm 0.18$ & 0.999 & 4.9 \\
\hline $\mathrm{Ch}+\mathrm{QA} 15-\mathrm{Ch} 50$ & $4.94 \pm 0.01$ & 0.997 & 2.4 \\
\hline $\mathrm{Ch}+\mathrm{QA} 20-\mathrm{Ch} 50$ & $4.88 \pm 0.04$ & 0.999 & 3.6 \\
\hline $\mathrm{Ch}+\mathrm{QA} 10-\mathrm{Ch} 50-\mathrm{SH}$ & $4.72 \pm 0.19$ & 0.998 & 6.7 \\
\hline $\mathrm{Ch}+\mathrm{QA}$ I5-Ch50-SH & $4.83 \pm 0.14$ & 0.997 & 4.5 \\
\hline $\mathrm{Ch}+\mathrm{QA} 20-\mathrm{Ch} 50-\mathrm{SH}$ & $4.87 \pm 0.23$ & 0.993 & 3.8 \\
\hline
\end{tabular}

Note: aBound fraction.

Abbreviations: 5-FU, 5-fluorouracil; SD, standard deviation; Ch, chitosan; QA, quaternary ammonium; NS, not significant. initial concentration. As expected, ${ }^{20}$ a straight line with a negative slope was obtained in all cases, the significance of which is shown by the relevant $r^{2}$ values $\left(r^{2} \geq 0.993\right)$. The abscissa intersection of each straight line gave the equilibrium concentration with the gel. This corresponded with the unbound-drug concentration in the hydrogel, from which the bound concentration could readily be calculated. From data in Table 3, it appears that the drug binding to $\mathrm{Ch}$ in the gel was much stronger than in the sol state and the partial substitution of $\mathrm{Ch}$ in the hydrogel by $\mathrm{Ch}$ derivatives caused a further increase in the drug-gel interaction. This increase was perhaps due to a stiffening of the polymer network that could correlate with the presence of fixed positive charges

Table 3 Results of phase-equilibration studies

\begin{tabular}{lll}
\hline Formulation & $\begin{array}{l}\text { Equilibrium } \\
\text { concentration in } \\
\text { PB } \times 1 \mathbf{1}^{2}(\mathbf{m g} / \mathbf{m L})\end{array}$ & $\begin{array}{l}\text { Bound } \\
\text { concentration } \\
(\%)\end{array}$ \\
\hline Ch & 16.1 & 83.9 \\
Ch + QA10-Ch60 & 6.1 & 93.9 \\
Ch + QA15-Ch60 & 6.5 & 93.5 \\
Ch + QA20-Ch60 & 6.7 & 93.3 \\
Ch + QA10-Ch60-SH & 7.9 & 92.1 \\
Ch + QA15-Ch60-SH & 6.7 & 93.3 \\
Ch + QA20-Ch60-SH & 7.1 & 92.9 \\
Ch + QA10-Ch50 & 8.5 & 91.5 \\
Ch + QA15-Ch50 & 5.5 & 94.5 \\
Ch + QA20-Ch50 & 4.6 & 95.4 \\
Ch + QA10-Ch50-SH & 15.7 & 84.3 \\
Ch + QA15-Ch50-SH & 12.8 & 87.2 \\
Ch + QA20-Ch50-SH & 12.9 & 87.1 \\
\hline Notes: & &
\end{tabular}

Notes: Initial concentration of $5-\mathrm{FU}$ in $\mathrm{TSOH} 1.25 \mathrm{mg} / \mathrm{mL}$. In all cases, $r^{2} \geq 0.993 ; n=6$.

Abbreviations: $\mathrm{PB}$, phosphate buffer; $\mathrm{Ch}$, chitosan; $\mathrm{QA}$, quaternary ammonium; 5-FU, 5-fluorouracil; TSOH, thermosensitive ophthalmic hydrogel. 
of Ch derivatives. This hypothesis is endorsed by the higher drug-gel interaction registered with the conjugate QA20Ch50. Indeed, in this polymer, the number of QA ions was higher than the other derivatives, ${ }^{10}$ where the structure of these conjugates is shown. A shielding action of thiols reduced this effect, as shown in Table 3.

A remarkable affinity of the mucoadhesive $\mathrm{TSOH}$ and NPs for 5-FU has been evidenced in the foregoing discussion. In vitro release data are still lacking to guide the selection of an NP-containing TSOH to be tested in vivo as a potential controlled ophthalmic 5-FU-delivery system.

\section{Drug release from NP-containing TSOH}

Data on drug release from $\mathrm{TSOH}$, obtained as described in the "Studies on 5-FU release from TSOH" section and plotted as percentage of released drug vs $\sqrt{ } \mathrm{t}$, yielded linear plots (not reported for clarity) with few zero intercepts (between $-2.66 \%$ and $1.18 \%$ ) and high $r^{2}$ values (between 0.97 and $0.99 ; n=6)$. This pattern in all cases fitted well a well-known model assuming release is entirely governed by drug diffusion in the releasing vehicle. The slope of each straight line is a useful parameter allowing comparison of the different cases on the basis of drug diffusivity in the hydrogel. In this light, an insight into the data listed in Table 4 shows that the substitution of some fractions of $\mathrm{Ch}$ in the gelling mixture with a QA-Ch derivative exerted some effects on the release characteristics of the $\mathrm{TSOH}$, depending on the particular derivative. A decreasing effect on the slope of the percentage-release-vs- $\sqrt{t}$ plot and on cumulative release in 5 hours was significant, with the $\mathrm{Ch}+\mathrm{QA} 15-\mathrm{Ch} 50$ and
Ch + QA20-Ch50 hydrogels containing medicated NPs. Also, it should be pointed out that in all cases the slopes of the $\sqrt{t}$ plots for the plain hydrogels were higher than those for the NP-containing ones. Considering that for a mucoadhesive vehicle, drug-vehicle binding is directly related, while drug diffusivity in a vehicle is inversely related to drug retention in the precorneal area, the data described so far encouraged us to choose the NP-containing Ch + QA20-Ch50 formulation for the in vivo tests. For this formulation, indeed, Tables 3 and 4 show the highest bound concentration and the lowest diffusivity-related parameters, respectively.

\section{In vivo tests}

When instilled in rabbit eyes, the ophthalmic drops were biocompatible and caused no apparent irritation signs, such as conjunctival/corneal edema and/or hyperemia. The relevant concentration-time profiles are shown in Figure 6, while the pharmacokinetic data are listed in Table 5.

The results of the in vivo tests shown in Figure 6 demonstrate the ability of the selected TSOH + NP system to increase bioavailability with respect to the control. This system produced maximum concentration and AUC values significantly higher than that relative to the reference. Note that the AUC value for the hydrogel obtained from plain $\mathrm{Ch}$ was significantly lower than that relative to the hydrogel containing the $\mathrm{Ch}$ derivative. This was probably due to the ability of the $\mathrm{Ch}+\mathrm{QA20-Ch50} \mathrm{formulation} \mathrm{to} \mathrm{control} \mathrm{the} \mathrm{release}$ of 5-FU. The AUC values shown in Table 5, relative to the $\mathrm{Ch}+\mathrm{QA} 20-\mathrm{Ch} 50$ hydrogels containing (or not) NPs, were not significantly different. However, the NP-containing hydrogel

Table 4 Characteristics of 5-FU release from plain or NP-containing TSOH

\begin{tabular}{|c|c|c|c|}
\hline TSOH composition & $\begin{array}{l}\text { Slope of } \sqrt{ } \mathrm{t} \text { plot for } \\
\text { release from plain } \\
\text { TSOH }(\% / \sqrt{ } \text { min })\end{array}$ & $\begin{array}{l}\text { Slope of } \sqrt{ } \mathrm{t} \text { plot for release } \\
\text { from NP-containing } \\
\text { TSOH }(\% / \sqrt{ } \text { min) }\end{array}$ & $\begin{array}{l}\text { Cumulative release } \\
\text { from NP-containing } \\
\text { TSOH at } 5 \text { hours (\%) }\end{array}$ \\
\hline $\mathrm{Ch}$ & $7.04 \pm 0.53$ & $4.72 \pm 0.16$ & $82.8 \pm 4.7$ \\
\hline $\mathrm{Ch}+\mathrm{QA} 10-\mathrm{Ch} 60$ & $7.03 \pm 0.16$ & $4.25 \pm 0.17$ & $79.6 \pm 3.9$ \\
\hline $\mathrm{Ch}+\mathrm{QAI} 5-\mathrm{Ch} 60$ & $6.77 \pm 0.18$ & $4.34 \pm 0.28$ & $78.7 \pm 3.8$ \\
\hline $\mathrm{Ch}+\mathrm{QA} 20-\mathrm{Ch} 60$ & $5.76 \pm 0.17$ & $4.62 \pm 0.24$ & $78.9 \pm 0.6$ \\
\hline $\mathrm{Ch}+\mathrm{QAI} 0-\mathrm{Ch} 60-\mathrm{SH}$ & $7.8 \pm 0.51$ & $4.47 \pm 0.23$ & $82.2 \pm 4.2$ \\
\hline $\mathrm{Ch}+\mathrm{QAI} 5-\mathrm{Ch} 60-\mathrm{SH}$ & $6.77 \pm 0.25$ & $4.78 \pm 0.24$ & $86 \pm 3.9$ \\
\hline $\mathrm{Ch}+\mathrm{QA20}-\mathrm{Ch} 60-\mathrm{SH}$ & $5.81 \pm 0.34$ & $4.3 I \pm 0.34$ & $76.2 \pm 2.9$ \\
\hline $\mathrm{Ch}+\mathrm{QA} 10-\mathrm{Ch} 50$ & $6.40 \pm 0.2$ & $4.45 \pm 0.36$ & $84.1 \pm 7.6$ \\
\hline $\mathrm{Ch}+\mathrm{QAI} 5-\mathrm{Ch} 50$ & $7.72 \pm 0.22$ & $3.67 \pm 0.36$ & $69.5 \pm 1.5$ \\
\hline $\mathrm{Ch}+\mathrm{QA20}-\mathrm{Ch} 50$ & $6.45 \pm 0.06$ & $3.34 \pm 0.32$ & $59.5 \pm 0.9$ \\
\hline $\mathrm{Ch}+\mathrm{QAI} 0-\mathrm{Ch} 50-\mathrm{SH}$ & $7.68 \pm 0.16$ & $4.98 \pm 0.35$ & $86.5 \pm 1.8$ \\
\hline $\mathrm{Ch}+\mathrm{QAI} 5-\mathrm{Ch} 50-\mathrm{SH}$ & $7.49 \pm 0.07$ & $4.62 \pm 0.54$ & $87.9 \pm 2.3$ \\
\hline $\mathrm{Ch}+\mathrm{QA} 20-\mathrm{Ch} 50-\mathrm{SH}$ & $6.54 \pm 0.2$ & $4.02 \pm 0.31$ & $78.4 \pm 3.8$ \\
\hline
\end{tabular}

Notes: $r^{2} \geq 0.997 ; \mathrm{n}=6$.

Abbreviations: 5-FU, 5-fluorouracil; NP, nanoparticle; TSOH, thermosensitive ophthalmic hydrogel; Ch, chitosan; QA, quaternary ammonium. 


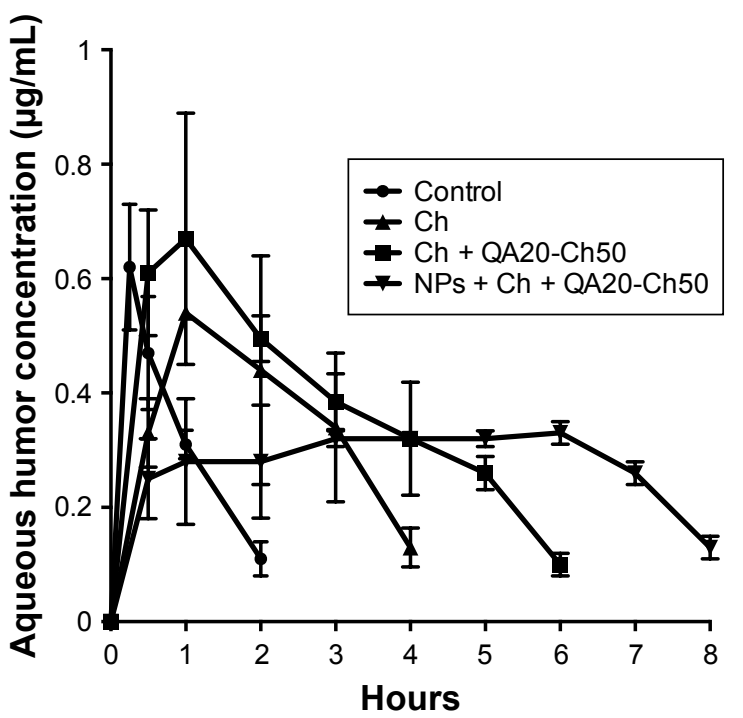

Figure 6 Pharmacokinetics in the aqueous following instillation of ophthalmic drops. Note: Each data point is the mean \pm standard deviation of at least six values obtained with different animals.

Abbreviations: $\mathrm{Ch}$, chitosan; QA, quaternary ammonium; NPs, nanoparticles.

showed a concentration plateau in the range $0.5-7$ hours. This pattern points to zero-order transcorneal absorption kinetics, which can be ascribed to controlled drug release from the selected NP-containing TSOH, perhaps accompanied by prolonged TSOH residence in the precorneal area, in turn due to the mucoadhesiveness of formulation components. Also, NP internalization by the corneal cells could be an important factor determining pharmacokinetics in the aqueous humor.

\section{Conclusion}

TSOH was successfully prepared from solutions of $\mathrm{Ch}$ and its mucoadhesive derivatives. The sol-gel transition occurred in the interval $30^{\circ} \mathrm{C}-35^{\circ} \mathrm{C}$. The TSOH and Ch-based NPs showed a remarkable affinity for 5-FU. Drug-binding and in vitro drug-release studies guided the selection of an NP-containing TSOH to make a potential controlled 5-FU-delivery system for ophthalmic application. Increased bioavailability of 5-FU in the aqueous with respect to the reference resulted

Table 5 Pharmacokinetic data obtained in vivo in rabbit eyes

\begin{tabular}{lllll}
\hline Formulation & $\mathbf{C}_{\max }$, & $\mathbf{T}_{\max }$, & $\mathbf{A U C} \mathbf{C}_{0-8}$, & AUC $_{\text {rel }}$ \\
& $\mu \mathrm{g} / \mathbf{m L}$ & hours & $\mu \mathrm{g} \cdot \mathbf{h} / \mathbf{m L}$ & \\
\hline Control & $0.62 \pm 0.1 \mathrm{I}$ & 0.25 & $0.62 \pm 0.1$ & - \\
Ch & $0.54 \pm 0.0 \mathrm{I}$ & $\mathrm{I}$ & $\mathrm{I} .42 \pm 0.38 *$ & 2.3 \\
Ch + QA20-Ch50 & $0.67 \pm 0.22$ & $\mathrm{I}$ & $2.32 \pm 0.36 *$ & 3.7 \\
NPs + Ch + QA20-Ch50 & - & - & $2.23 \pm 0.39 *$ & 3.6 \\
\hline
\end{tabular}

Note: $* p<0.05$.

Abbreviations: $C_{\max }$, maximum concentration; $T_{\max }$, time to $C_{\max } ; A \cup C_{0-8}$, area under the curve, $0-8$ hours; $A \cup C_{\text {rel }}$, relative AUC; $C h$, chitosan; $Q A$, quaternary ammonium; NPs, nanoparticles. from in vivo experiments in rabbit eyes. The NP-containing hydrogels mediated to a zero-order 5-FU absorption, leading to a time-constant anticancer concentration in the aqueous for up to 7 hours from instillation. The TSOH and NPs coupled their properties to produce an interesting sustained-release formulation for ophthalmic application.

\section{Disclosure}

The authors report no conflicts of interest in this work. The preliminary data of this research paper were presented at the 10th World Meeting on Pharmaceutics, Biopharmaceutics and Pharmaceutical Technology, April 4-7, 2016, in Glasgow.

\section{References}

1. Le Bourlais C, Acar L, Zia H, Sado PA, Needham T, Leverge R. Ophthalmic drug delivery systems-recent advances. Prog Retin Eye Res. 1998;17:35-58.

2. Yasukawa T, Ogura Y, Tabata Y, Kimura H, Wiedemann P, Honda Y. Drug delivery systems for vitreoretinal diseases. Prog Retin Eye Res. 2004;23:253-281.

3. Sahoo SK, Dilnawaz F, Krishnakumar S. Nanotechnology in ocular drug delivery. Drug Discov Today. 2008;13:144-151.

4. Berger J, Reist M, Chenite A, Felt-Baeyens O, Mayer JM, Gurny R. Pseudo-thermosetting chitosan hydrogels for biomedical application. Int J Pharm. 2005;288:197-206.

5. Fernández-Ferreiro A, González Barcia M, Gil-Martínez M, et al. In vitro and in vivo ocular safety and eye surface permanence determination by direct and magnetic resonance imaging of ion-sensitive hydrogels based on gellan gum and kappa-carrageenan. Eur J Pharm Biopharm. 2015;94:342-351.

6. Zhou HY, Jiang LJ, Cao PP, Li JB, Chen XG. Glycerophosphate-based chitosan thermosensitive hydrogels and their biomedical applications. Carbohydr Polym. 2015;117:524-536.

7. Nagarwal R C, Kumar R, Dhanawat M, Pandit JK. Modified PLA nano in situ gel: a potential ophthalmic drug delivery system. Colloids Surf B Biointerfaces. 2011;86:28-34.

8. Lou J, Hu W, Tian R, et al. Optimization and evaluation of a thermoresponsive ophthalmic in situ gel containing curcumin-loaded albumin nanoparticles. Int J Nanomedicine. 2014;9:2517-2525.

9. Chenite A, Chaput C, Wang D, et al. Novel injectable neutral solutions of chitosan form biodegradable gels in situ. Biomaterials. 2000; 21:2155-2161.

10. Zambito Y, Felice F, Fabiano A, Di Stefano R, Di Colo G. Mucoadhesive nanoparticles made of thiolated quaternary ammonium chitosan crosslinked with hyaluronan. Carbohydr Polym. 2013;92:33-39.

11. Zambito Y, Di Colo G. Thiolated quaternary ammonium-chitosan conjugates for enhanced precorneal retention, transcorneal permeation and intraocular absorption of dexamethasone. Eur J Pharm Biopharm. 2010;72:194-199.

12. Nagarwal RC, Singh PN, Kant S, Maiti P, Pandit JK. Chitosan nanoparticles of 5-fluorouracil for ophthalmic delivery: characterization, in vitro and in vivo study. Chem Pharm Bull (Tokyo). 2011;59:272-278.

13. Zambito Y, Baggiani A, Carelli V, Serafini MF, Di Colo G. Matrices for site-specific controlled-delivery of 5-fluorouracil to descending colon. J Control Release. 2005;102:669-677.

14. Clausen AE, Bernkop-Schnürch A. In vitro evaluation of the permeationenhancing effect of thiolated polycarbophil. J Pharm Sci. 2000; 89:1253-1261.

15. Clausen AE, Bernkop-Schnürch A. Thiolated carboxymethylcellulose: in vitro evaluation of the permeation enhancing effect on peptide drugs. Eur J Pharm Biopharm. 2001;51:25-32. 
16. Di Colo G, Zambito Y, Zaino C, Sansò M. Selected polysaccharide at comparison for their mucoadhesiveness and effect on precorneal residence of different drugs in the rabbit model. Drug Dev Ind Pharm. 2009;35:941-949.

17. Uccello-Barretta G, Nazzi S, Balzano F, et al. Enhanced affinity of ketotifen toward tamarind seed polysaccharide in comparison with hydroxyethylcellulose and hyaluronic acid: a nuclear magnetic resonance investigation. Bioorg Med Chem. 2008;16:7371-7376.

18. Bottari F, Di Colo G, Nannipieri E, Saettone MF, Serafini MF. Evaluation of a dynamic permeation technique for studying drug-macromolecule interaction. J Pharm Sci. 1975;64:946-949.

19. Zambito Y, Pedreschi E, Di Colo G. Is dialysis a reliable method for studying drug release from nanoparticulate systems? A case study. Int J Pharm. 2012;434:28-34.

20. Di Colo G, Zambito Y. A study of release mechanisms of different ophthalmic drugs from erodible ocular inserts based on poly(ethylene oxide). Eur J Pharm Biopharm. 2002;54:193-199.

21. Lallemand F, Furrer P, Felt-Baeyens O, et al. A novel water-soluble cyclosporine A prodrug: ocular tolerance and in vivo kinetics. Int J Pharm. 2005;295:7-14.

22. Zambito Y, Zaino C, Di Colo G. Effects of N-trimethylchitosan on transcellular and paracellular transcorneal drug transport. Eur J Pharm Biopharm. 2006;64:16-25.

23. Schoenwald RD, Harris RG, Turner D, Knowles W, Chien DS. Ophthalmic bioequivalence of steroid/antibiotic combination formulations. Biopharm Drug Dispos. 1987;8:527-548.
24. Tamba BI, Dondas A, Leon M, et al. Silica nanoparticles: preparation, characterization and in vitro/in vivo biodistribution studies. Eur JPharm Sci. 2015;71:46-55.

25. Khan MS, Vishakante GD, Bathool A. Development and characterization of pilocarpine loaded Eudragit nanosuspensions for ocular drug delivery. J Biomed Nanotechnol. 2013;9:124-131.

26. Singh KH, Shinde UA. Chitosan nanoparticles for controlled delivery of brimonidine tartrate to the ocular membrane. Pharmazie. 2011;66: 594-599.

27. Pucci A, Bizzarri R, Ruggeri G. Polymer composites with smart optical properties. Soft Matter. 2011;7:3689-3700.

28. Pucci A, Signori F, Bizzarri R, Bronco S, Ruggeri G, Ciardelli F. Threshold temperature luminescent indicators from biodegradable poly (lactic acid)/poly(butylene succinate) blends. J Mater Chem. 2010;20: 5843-5852.

29. Uccello-Barretta G, Nazzi S, Zambito Y, Di Colo G, Balzano F, Sansò M. Synergistic interaction between TS-polysaccharide and hyaluronic acid: implications in the formulation of eye drops. Int J Pharm. 2010;395: 122-131.

30. Fabiano A, Chetoni P, Zambito Y. Mucoadhesive nano-sized supramolecular assemblies for improved pre-corneal drug residence time. Drug Dev Ind Pharm. 2015;41:2069-2076.
International Journal of Nanomedicine

\section{Publish your work in this journal}

The International Journal of Nanomedicine is an international, peerreviewed journal focusing on the application of nanotechnology in diagnostics, therapeutics, and drug delivery systems throughout the biomedical field. This journal is indexed on PubMed Central, MedLine, CAS, SciSearch $®$, Current Contents $\AA /$ Clinical Medicine,

\section{Dovepress}

Journal Citation Reports/Science Edition, EMBase, Scopus and the Elsevier Bibliographic databases. The manuscript management system is completely online and includes a very quick and fair peer-review system, which is all easy to use. Visit http://www.dovepress.com/ testimonials.php to read real quotes from published authors. 